\title{
Brownout Simulations of Model-Rotors In Ground Effect
}

\author{
$F$. Rovere ${ }^{1, *}$, G.N. Barakos ${ }^{1, \dagger}$, and R. Steij $1^{1, *}$ \\ ${ }^{1}$ CFD Laboratory School of Engineering, University of Glasgow
}

\begin{abstract}
In this work computational fluid dynamics is used to validate experimental results for a two-bladed small rotor In Ground Effect conditions. The paper focuses on the evaluation and prediction of the rotor outwash generated in ground effect. Time-averaged outflow velocities are compared with experimental results, and the simulated flow field is used for safety studies using the PAXman model and particle tracking methods. The aircraft weights have been studied, evaluating scaling factors to define how helicopter weight can affect the outflow forces and the particle paths. Results show how the wake generated by heavier helicopters can lead to stronger forces on ground personnel and push the particles farther away from the rotor.
\end{abstract}

\section{Nomenclature}

\section{Acronyms}

CFD Computational Fluid Dynamics

DVE Degraded Visual Environment

IGE In Ground Effect

MTOW Maximum Take-Off Weight

MUSCL Monotone Upstream Centered Schemes for Conservations Laws OGE Out of Ground Effect

\footnotetext{
* PhD Student - federico.rovere@glasgow.ac.uk

$\dagger$ Professor - george.barakos@glasgow.ac.uk

† Senior Lecturer - rene.steijl@glasgow.ac.uk
} 
PIV Particle Image Velocimetry

\section{Greek}

$\mathrm{N}$ Kinematic viscosity $\mathrm{m} 2 / \mathrm{s}$

$\Psi \quad$ Local azimuth angle, deg.

$\rho \quad$ Density, $\mathrm{kg} / \mathrm{m} 3$

$\theta_{75}$ Collective pitch at three-quarter radius, deg.

\section{Latin}

u Flow field velocity vector, $\mathrm{m} / \mathrm{s}$

up Particle velocity vector, $\mathrm{m} / \mathrm{s}$

a Speed of sound, $\mathrm{m} / \mathrm{s}$

$\mathbf{a}_{\mathrm{p}}$ Particle acceleration, $\mathrm{m} / \mathrm{s}^{2}$

B Ballistic coefficient, $\mathrm{kg} / \mathrm{m}^{2}$

c Blade chord, $\mathrm{m}$

$\mathrm{C}_{\mathrm{D}}$ Drag coefficient

$\mathrm{C}_{\mathrm{Q}} \quad$ Rotor torque coefficient, $\mathrm{C}_{\mathrm{Q}}=\mathrm{Q} /\left(0.5 \mathrm{Q} \mathrm{V}_{\text {tip }}^{2} \mathrm{~S}_{\text {rotor }} \mathrm{R}\right)$

$\mathrm{C}_{\mathrm{T}} \quad$ Rotor thrust coefficient, $\mathrm{C}_{\mathrm{T}}=\mathrm{T} /\left(0.5 \mathrm{Q} \mathrm{V}_{\text {tip }}^{2} \mathrm{~S}_{\text {rotor }}\right)$

$d_{p} \quad$ Rotor diameter, $m$

g Gravity acceleration, $\mathrm{m} / \mathrm{s}^{2}$

$\mathrm{h}_{\text {PAXman }} \quad$ PAXman height scaled, $\mathrm{m}$

M Mach number, $\mathrm{M}=\mathrm{V}_{\text {tip }} / \mathrm{a}$

$\mathrm{m}_{\mathrm{p}} \quad$ Mass particle, $\mathrm{kg}$

$\mathrm{N}_{\mathrm{b}} \quad$ Number of blades

Q Rotor torque, $\mathrm{N} \cdot \mathrm{m}$

$\mathrm{R} \quad$ Rotor radius, $\mathrm{m}$

r Radial coordinate along blade span, $\mathrm{m}$ 
Re Reynolds number, $\operatorname{Re}=\mathrm{V}_{\text {tip }} \mathrm{c} / \mathrm{v}$

$\mathrm{S}_{\mathrm{p}} \quad$ Frontal particle area, $\mathrm{m}^{2}$

$\mathrm{S}_{\text {rotor }}$ Rotor disk area, $\mathrm{m}^{2}$

T Rotor thrust, $\mathrm{N}$

U Velocity x-component, $\mathrm{m} / \mathrm{s}$

u* Friction velocity, $\mathrm{m} / \mathrm{s}$

V Velocity y-component, $\mathrm{m} / \mathrm{s}$

$\mathrm{v}_{\mathrm{i}} \quad$ Hover induced velocity,

$\mathrm{v}_{\max }$ Highest value of radial velocity

$\mathrm{V}_{\mathrm{rad}}=\mathrm{U} \cos (\Psi)+\mathrm{V} \sin (\Psi), \mathrm{m} / \mathrm{s}$

\section{Super and sub scripts}

fs Full scale

p Particle

ss Small scale

tip Blade tip value

$\infty \quad$ Freestream value

\section{Introduction}

The flow fields generated by rotors operating In Ground Effect (IGE) are complex and unsteady and may also interact with the ground plane. The result of this interaction is the transition of the rotor induced flow from vertical (downwash) to radial flow (outwash). This flow field can be the source of risks for ground personnel, equipment and landscape due to the forces generated by high outflow velocities. Furthermore, in case of brownout or whiteout the flow field can interact with a loose sediment bed, uplifting particles all around the aircraft. The prediction of the outwash is fundamental for safety of helicopter IGE operations. The downwash typically affects activities directly under the aircraft (like search and rescue operations), while the outwash impacts on the surrounding environment like people, equipment and structures during landing and take-off. As already mentioned, brownout and whiteout may occur when a rotorcraft is operating in ground proximity and they are due to the interaction of rotor wake with the particles of a loose sediment bed such as sand, snow etc. This interaction can eventually cause the uplift of particles from the ground and their entrainment into the air flow. When operating in desert areas or in snowy regions, the number of particles entrained can become extremely high creating a cloud around the rotorcraft. The main effects of this cloud of particles moving around the aircraft are on the pilot's visual 
environment and on the rotorcraft structure and equipment. The lack of pilot visibility defines brownout and whiteout as Degraded Visual Environment (DVE) conditions. In recent years, efforts have been made to help pilots in these situations, developing sensors and advanced cockpit displays. Dynamic rollover and collisions with objects are common accidents due to the lack of visibility [1]. In [2] NATO statistics are given about mishaps due to brownout or whiteout: US Air force lost 30 special operation aircraft and 60 crew members lost their lives during landing in desert environments since 1990. In the same report authors specify that brownout cost to US services $\$ 100 \mathrm{M} / \mathrm{yr}$. Other NATO members experienced similar statistics. UK had 24 brownout mishaps in the period 2005-2009. The German defence force had more than 30 accidents due to dust and snow. In [3], the authors refer that the occurrence of brownout is the most common cause of human factor mishaps during military operations. In the present work, computational fluid dynamics (CFD) analysis of a micro two-bladed rotor operating IGE is compared with experimental results. The test case simulated was experimentally investigated at the University of Maryland by Lee et al. [4]. The flow field data was obtained by 2D Particle Image Velocimetry (PIV), while the rotor performance was measured by a micro mass balance. Once the CFD analysis was complete, the outflows produced were used to evaluate the forces produced on ground personnel, properly scaled, and using the PAXman model, and particle tracking. To obtain realistic full-scale scenarios, a scaling factor has been applied to velocities using three different aircraft, categorized in terms of weight. After this, safe zones are defined where it was possible to consider the outflow forces and the presence of the particles as negligible. Existing safety separation criteria can then be tested. A possible separation distance is suggested by FAA for wake encounters [5] [6]. In that case, a distance of 3 rotor diameters is suggested to allow the dissipation of the wake generated by a rotor in hover or taxiing. Investigations are conducted to verify if the same distance can be considered safe for forces due to the outflow acting on ground personnel and for the presence of particles in the area that can spoil the pilot view of a near aircraft or hit people operating inside the 3 rotor diameters area. All CFD simulations have been performed using the HMB3 (Helicopter Multi-Block) CFD solver of Glasgow University.

\section{CFD solver}

The HMB3 (Helicopter Multi-Block) [7] [8] is the solver used for CFD calculations in this work. It solves the Unsteady Reynolds Averaged Navier-Stokes equations (URANS) in integral form with ALE formulation (Arbitrary Lagrangian Eulerian) formulation for timedependent domains (moving boundaries). URANS equations are discretised using a cellcentred finite volume approach on a multiblock structured grid. HMB uses the Osher [9] and Roe [10] approximate Riemann solvers to evaluate the convective fluxes, the viscous terms are discretized using second order central differencing. Third order accuracy in space is provided by The Monotone Upstream Centred Schemes for Conservation Laws (MUSCL) [11]. An implicit dual time stepping methods is employed to perform the temporal integration. Oversets grids (used in this work) [12] and sliding plane [13] methods are available in HMB to allow for the relative motion between mesh components, representing ground and rotor blades.

\subsection{PAXman model}

The PAXman model was originally developed for military personnel and it is used to compute wind forces on people operating in proximity to the rotor. It is based on the projection of a crouching $6 \mathrm{ft}$ tall person immersed in the outwash. The details of the geometry 
of the PAXman model are shown in figure 1, as reported in [14] and [15]. To be comparable with the small rotor size, the PAXman height is scaled with the rotor radius for three different full-scale rotors, chosen to represent three different weight categories: light, medium and heavy. Their technical data are listed in table 2.

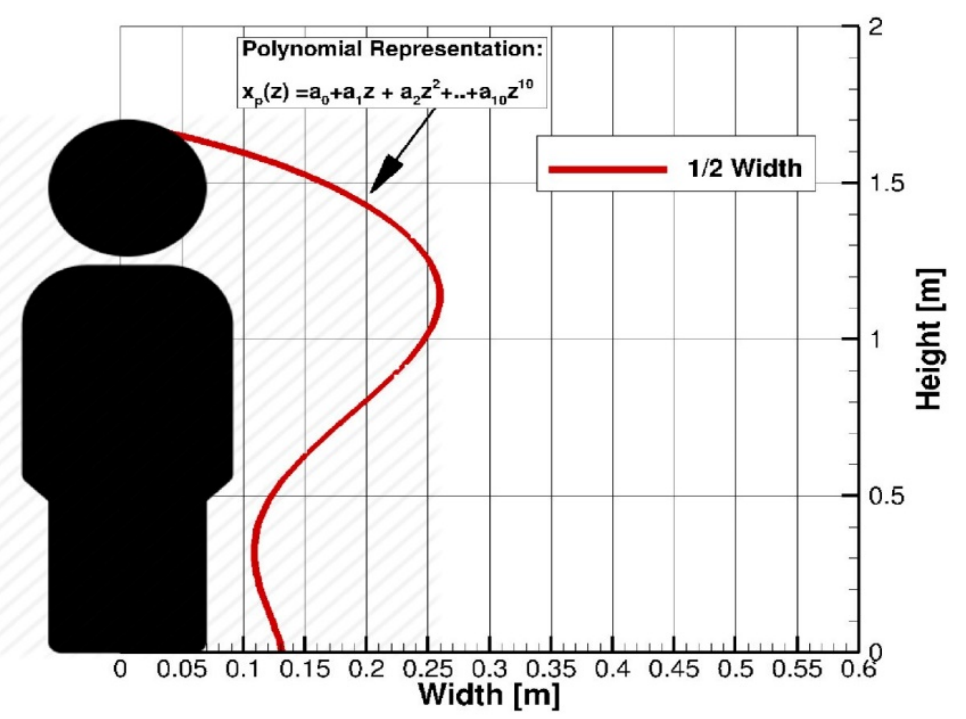

Figure 1: PAXman model [15]

Table 1: Polynomial coefficients used in figure [1]

\begin{tabular}{|l|l|}
\hline a0 & $4.30939 \mathrm{e}-01$ \\
\hline a1 & $-4.63972 \mathrm{e}-02$ \\
\hline a2 & $-1.39649 \mathrm{e}-01$ \\
\hline a3 & $1.37545 \mathrm{e}-01$ \\
\hline a4 & $1.37545 \mathrm{e}-01$ \\
\hline a5 & $-5.49253 \mathrm{e}-04$ \\
\hline a6 & $2.21653 \mathrm{e}-04$ \\
\hline a7 & $-4.18444 \mathrm{e}-05$ \\
\hline a8 & $1.45194 \mathrm{e}-05$ \\
\hline a9 & $-7.80009 \mathrm{e}-08$ \\
\hline a10 & $-1.89822 \mathrm{e}-07$ \\
\hline
\end{tabular}

Table 2 Helicopters technical data [16]

\begin{tabular}{|c|c|c|c|c|}
\hline Category & MTOW $(\mathrm{kg})$ & $\mathrm{R}(\mathrm{m})$ & $\mathrm{V}_{\text {tip }}(\mathrm{m} / \mathrm{s})$ & $\mathrm{C}_{\mathrm{T}}$ \\
\hline Light & 3000 & 5.5 & 220 & 0.009 \\
\hline Medium & 8000 & 8.1 & 216 & 0.015 \\
\hline Heavy & 11000 & 8.1 & 220 & 0.0176 \\
\hline
\end{tabular}

The PAXman height has been scaled with the rotor radius, giving of $h_{P A X m a n}=0.3 \mathrm{R}$ for the lighter aircraft, while for the medium and the heavy cases the result is the same, due to 
the similar size of the rotor radius, which lead to $h_{\text {PAXman }}=0.2 \mathrm{R}$. Using the reference area expressed by the polynomial representation of figure 1 , the distribution of the force produced by the outwash on the ground personnel is computed as proposed in [14]. To obtain comparable forces with the safety criteria, velocities are scaled using the blade tip velocity, listed in table 2. For this first scaling is necessary, to obtain velocities expressed in $\mathrm{m} / \mathrm{s}$, that can be used, later, to compute the PAXman forces in N. However, a second scaling is necessary to take into account the difference in $\mathrm{C}_{\mathrm{T}}$ of the three different cases. A common reference value for outflow velocities is the dimensionless hover induced velocity $\lambda_{i}$, expressed as $\lambda_{\mathrm{i}}=\sqrt{\left(C_{T}\right)} / 2$. The scaled rotor thrust coefficient obtained by the simulation of the micro rotor is $\mathrm{C}_{\mathrm{T}}{ }^{\mathrm{ss}}=0.03$, while the $\mathrm{C}_{\mathrm{T}}{ }^{\mathrm{fs}}$ can be obtained by the data, considering the aircraft in hover flight, and thrust equal to the weight, that in this case is considered the maximum at take-off. $\mathrm{W}_{\text {MTOW }}, \mathrm{V}_{\text {tip }}$, and $\mathrm{S}_{\text {rotor }}$ or are specified in table 2, while for the acceleration of gravity and for the air density the following values have been assumed: $g=9.81 \mathrm{~m} / \mathrm{s}^{2}$ and $\rho_{\text {air }}=$ $1.225 \mathrm{~kg} / \mathrm{m}^{3}$

$$
C_{T}=\frac{2 W_{M T O W}}{0.5 \rho V_{\text {tip }}^{2} S_{\text {rotor }}}
$$

It is possible, taking into account the effect of the different thrust coefficient to scale the velocities using the ratio of hover induced velocity between full-scale and small-scale cases. The scaling factor obtained is $\sqrt{C_{T}^{S S} / C_{T}^{f s}}$. This way, it is possible to estimate the outflow velocities generated by a full-scale rotor operating at the same high values of thrust coefficient of the scaled rotor. According to [14] and [15] the caution zone starts when the force acting on the PAXman is more than $80 \mathrm{lbf}(335 \mathrm{~N})$, and the hazard zone is defined after $115 \mathrm{lbf}(510 \mathrm{~N})$. The distribution of the force over the body is calculated as: $f_{P A X m a n}=$ $0.5 \rho_{\text {air }} V 2_{\text {rad }} x$ where $\rho_{\text {air }}$ is density of the air, $\mathrm{V}_{\text {rad }}$ the radial velocity and $\mathrm{x}$ is the horizontal coordinate of the PAXman model. The total force is the integral of the distribution of the force over the height of the PAXman model.

$$
F_{P A X \operatorname{man}}=\int_{h_{P A X \operatorname{man}}} f_{P A X \operatorname{man}} d x
$$

Using this model, it is possible to calculate the force distribution acting on a human body at a specific radial station.

\subsection{Particle tracking}

Brownout and whiteout are due to the presence in the flow field of particles. The former involves sand, and the latter snow. However, other kinds of particles can be involved such as rain, ice and even small rocks. To properly simulate the behaviour of them in the flow field it is necessary model their motion. There are basically two approaches for the numerical simulation of dispersed phases, and they can be categorized into Lagrangian tracking or Eulerian modelling approaches. In the Lagrangian approach, the particles (or parcels of particles) are tracked through the field and the local cloud properties are defined by their properties as they pass the point in the field. For methods that involve this approach the motion of the particles is tracked solving the Newton's second law. Important works in Lagrangian frame of reference are [17] [18]. In the case of Eulerian methods, the properties of the particles are assumed to be continuous within the field. Thus, differential conservation equations are written, discretised, and the solution of these gives the properties of the cloud. [19] [20]. For particle tracking, an in-house software has been used. The particles are driven by the flow field velocities and their positions in time are obtained by integrating their 
equations of motion. The integration method used is a fourth order Runge-Kutta, and the equation for particle tracking acceleration is.

$$
\boldsymbol{a}_{p}=\frac{0.5 \rho_{\text {air }}\left(\boldsymbol{u}-\boldsymbol{u}_{\boldsymbol{p}}\right)\left\|\boldsymbol{u}-\boldsymbol{u}_{\boldsymbol{p}}\right\|}{B}-\mathbf{g}
$$

Where $\mathbf{a}_{\mathrm{p}}$ is the acceleration of the particle, $\mathbf{u}_{\mathrm{p}}$ is its velocity, $\mathbf{u}$ is the velocity of the flow field in the position of the particle and B is the ballistic coefficient, $B=m_{p} /\left(S_{p}-C_{D}\right)$. Here $\mathrm{m}_{\mathrm{p}}$ is the particle mass, $\mathrm{S}_{\mathrm{p}}$ is the particle frontal area (particles are assumed spherical) and $\mathrm{C}_{\mathrm{D}}$ is the particle drag coefficient, and finally $\mathbf{g}$ is acceleration of gravity. The particle proprieties used in this work are listed in table 3, they reflect the size and the density of particles used to simulate brownout in experimental and computational works [21] [22] [23].

Table 3 Properties of particles used in this work.

\begin{tabular}{|c|c|c|c|}
\hline$\rho_{\mathrm{p}}\left(\mathrm{kg} / \mathrm{m}^{3}\right)$ & $\mathrm{d}_{\mathrm{p}}(\mu \mathrm{m})$ & $\mathrm{C}_{\mathrm{D}}$ & $\mathrm{B}$ \\
\hline 2650 & 9 & 1.048 & 0.03 \\
\hline
\end{tabular}

\section{Computations and safety considerations}

Previous works show the full validation of the current methods [24]. Here a brief summary of the work is presented. IGE configuration the rotor was modelled using two overset grids, and the ground was modelled imposing no-slip and no-penetration conditions. The full rotor domain was computed as unsteady. Comparisons in terms of performance are given in figure 2 and show small differences with of the experiments of [4]. The thrust coefficient shows results very close to the result proposed by Lee et al. [4]. The increment of thrust coefficient IGE is about $12 \%$ with respect to the OGE case. The result of the power coefficient is slightly higher $\mathrm{C}_{\mathrm{Q}}{ }^{\mathrm{IGE}} / \mathrm{C}_{\mathrm{Q}}{ }^{\mathrm{OGE}}=1.02$. However, it can be considered that the power between OGE and IGE is fairy than what expected constant, with a small increment in the thrust coefficient, as expected. Similar results in terms of $\mathrm{C}_{\mathrm{Q}}^{\mathrm{IGE} /} \mathrm{C}_{\mathrm{Q}}{ }^{\mathrm{OGE}}$ have been obtained by Karla et al. in [25] for the same test case.

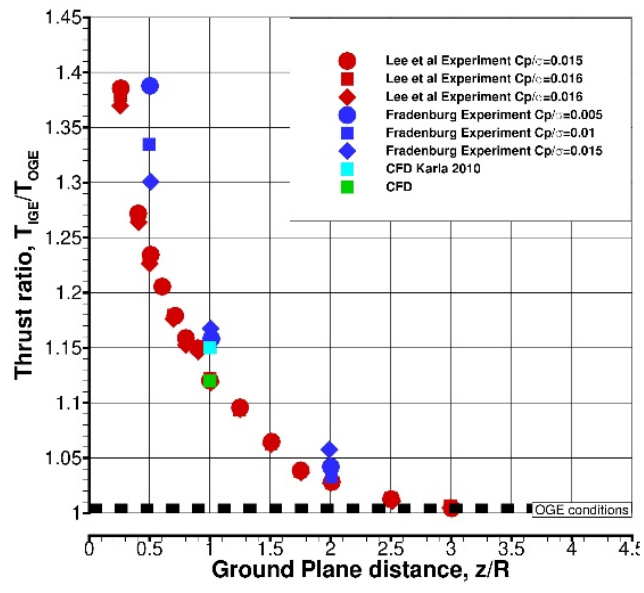

(a) $\mathrm{C}_{\mathrm{T}}{ }^{\mathrm{IGE}} / \mathrm{C}_{\mathrm{T}}{ }^{\mathrm{OGE}}$

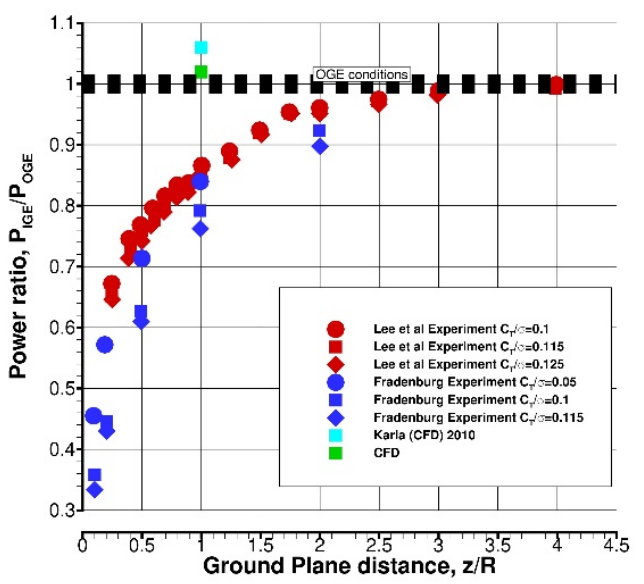

(b) $\mathrm{CQ}^{\mathrm{IGE} /} \mathrm{CQ}^{\mathrm{OGE}}$

Figure. 2: Experimental and CFD results for the trust (left) and power (right) ratios IGE. The rotor was operating at $\theta_{75}=12 \mathrm{deg}, \mathrm{Re}_{\mathrm{tip}}=35000$ and $\mathrm{M}_{\mathrm{tip}}=0.08$. Experimental data taken from [4] and additional CFD data from [25]. 
In figure 3, the rotor wake is presented as (b) iso-surfaces of Q-criterion and (a) contours of vorticity magnitude. The $\mathrm{Q}$ criterion is related to the velocity gradient tensor, ant its isosurfaces are a good indicator of how vortical flow may be [26]. As expected, the wake, reaches the ground and expands. The presence of tip vortices and vortex sheets are also expected

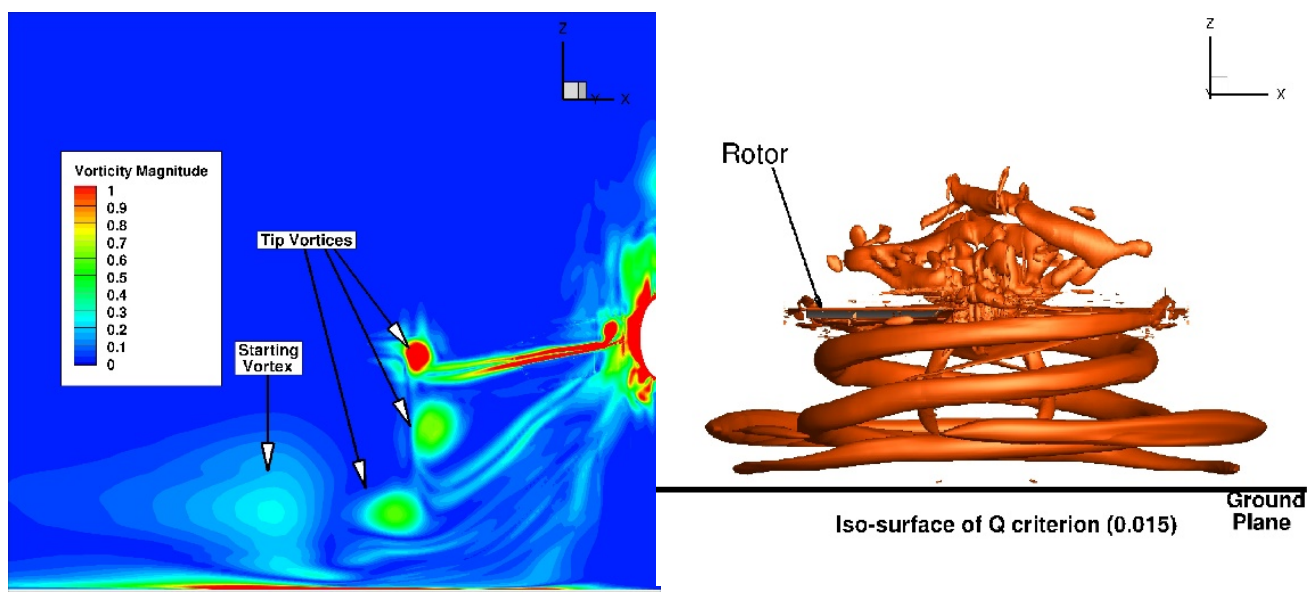

(a) Vorticity

(b) Q-criterion isosurfaces

Figure. 3 Wake Visualization, for the IGE case. The rotor operating at $\theta_{75}=12 \mathrm{deg}, \mathrm{Re}_{\mathrm{tip}}=35000$, $\mathrm{M}_{\text {tip }}=0.08, \mathrm{~h} / \mathrm{R}=1$ and $\mathrm{C}_{\mathrm{T}}=0.03$.

In figure 4 the time-averaged radial velocities are shown in comparison with experiments. The time-averaged outflow has been scaled using two different velocities. The left side of figure 4 shows results scaled with the reference hover induced velocity (as proposed by Lee et al. [4]), while on the right side of figure 4 the same results are scaled as a jet, using the peak radial velocity $\mathrm{v}_{\max }$. Finally, the dashed line represents the PAXman model height. The time-averaged velocities show good agreement with experiments in terms of maximum radial velocity and outflow distribution for both scaling methods, figure 4 (a) (b), (c) and (d), near the rotor. For higher radial positions, the peak velocity is underestimated, and its position is higher with respect to the ground, with some differences over the height distribution shown in figure 4 (e). This could be due to the few rotor revolutions performed in the simulation. 


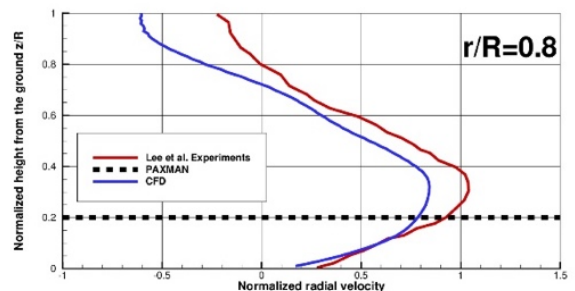

(a) $\mathrm{V}_{\mathrm{rad}} / \lambda_{\mathrm{i}}$ at $\mathrm{r} / \mathrm{R}=0.8$

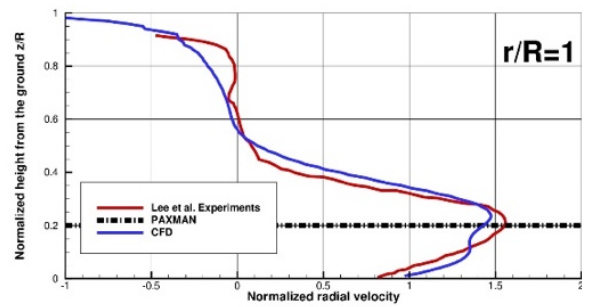

(c) $\mathrm{V}_{\mathrm{rad}} / \lambda_{\mathrm{i}}$ at $\mathrm{r} / \mathrm{R}=1.0$

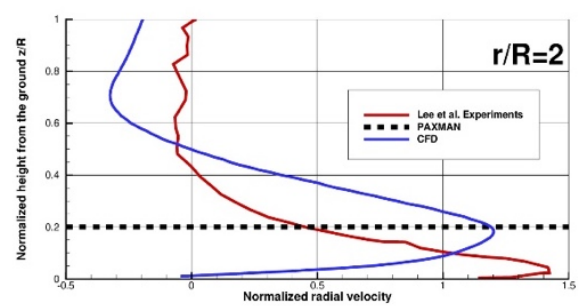

(e) $\mathrm{V}_{\mathrm{rad}} / \lambda_{\mathrm{i}}$ at $\mathrm{r} / \mathrm{R}=2.0$

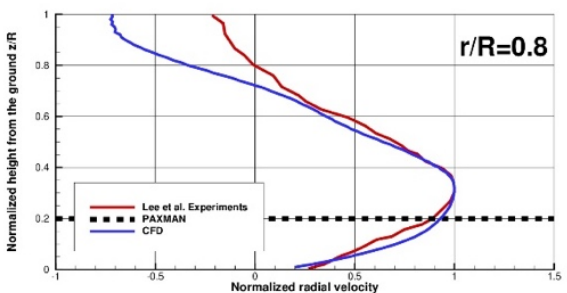

(b) $\mathrm{V}_{\mathrm{rad}} / \mathrm{V}_{\max }$ at $\mathrm{r} / \mathrm{R}=0.8$

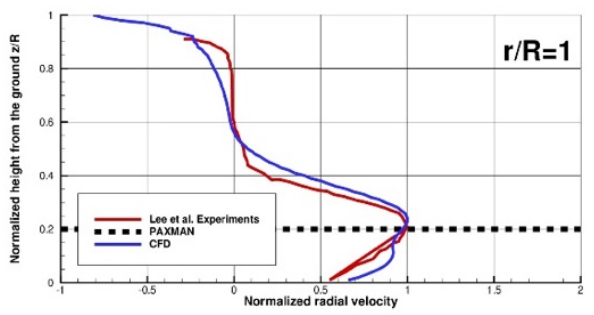

(d) $\mathrm{V}_{\mathrm{rad}} / \mathrm{V}_{\max }$ at $\mathrm{r} / \mathrm{R}=1.0$

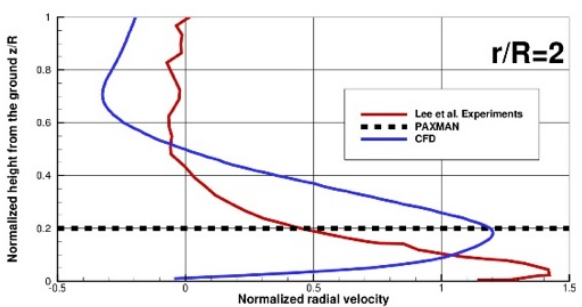

(f) $\mathrm{V}_{\mathrm{rad}} / \mathrm{V}_{\max }$ at $\mathrm{r} / \mathrm{R}=2.0$

Figure. 4 Experimental and CFD time-averaged outflow velocity profiles at different radial positions. The rotor was operating $\theta_{75}=12 \mathrm{deg}, \mathrm{Re}_{\mathrm{tip}}=35000 \mathrm{M}_{\mathrm{tip}}=0.08$ and $\mathrm{C}_{\mathrm{T}}=0.03$. Left column results are scaled with induced hover velocity, while the right column results are scaled with jet-scaled velocity. Experimental data taken from [4].

Figure 5 (a) shows the total forces acting on a scaled human body at different distances by the outflow produced by the rotor. As mentioned before, the forces have been computed scaling the velocities with the $\mathrm{V}$ tip of full-scale aircraft, to obtain more realistic values and compare them with the safety threshold suggested for civilian and military operations. The forces for all three aircraft reach their peaks at a radial distance between $1.25 \mathrm{R}$ and $1.5 \mathrm{R}$, but they weaken for larger radial stations. This result is due to the outflow distribution at different radial stations, with the higher velocities occurring around $1.5 \mathrm{R}$. As expected, the strongest force is produced by the aircraft with higher $\mathrm{C}_{\mathrm{T}}$, which is the heaviest helicopter taken into account. The outflow forces produced by the lightest helicopter are small in comparison to the other two helicopters and also with respect to the safety thresholds suggested by the PAXman method. For the other two cases, the forces exceed the caution limit, but do not reach the danger zone threshold. This result is in agreement with other works, such as in [15] where the PAXman forces of a V-22 have been computed starting from an experimental outflow survey. This reference involved several configurations of disk loading and it has been 
shown that higher values of disk loading result in forces that reached a peak of $600 \mathrm{~N}$. In all three cases, after $2 \mathrm{R}$, the force drops quickly. This is due to the outflow velocities that drops away from the rotor. After 3R the forces are almost negligible based on the PAXman model. Figure 5 (b), (c) and (d) show the distributions of the forces over the PAXman height at different radial stations for the three helicopters. For all three cases, at early distance from the rotor the force is strongest at a specific PAXman height (corresponding to the PAXman chest). This result is due to the shape of the outwash distribution and the larger blocking area at the chest level. This result is in accordance with other experiments. In [14] the force peaks were around the middle of the human body, however the experiments performed by Silva et al., were full-scale. Figure 5 (b) shows the results for the light aircraft. Here the PAXman height is higher with respect to the other two cases. This is due to the smaller size of the rotor blade of the lightest helicopter. Furthermore, the forces at every radial distance are smaller respect to heavier cases. This is in agreement with what is shown in figure 5 (a), where the lightest produces weaker forces in comparison to the medium and the heavy.

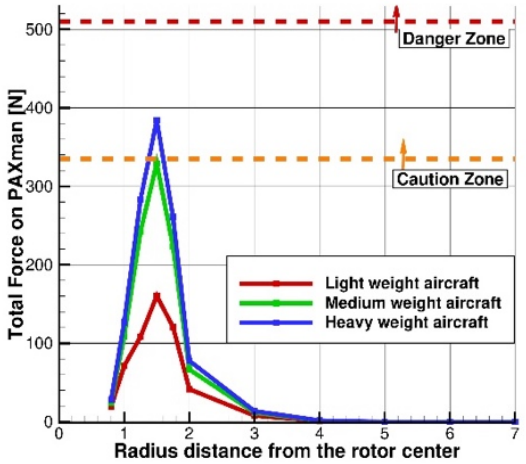

(a) Total radial force $F_{\text {PAXman }}$ for the three helicopters

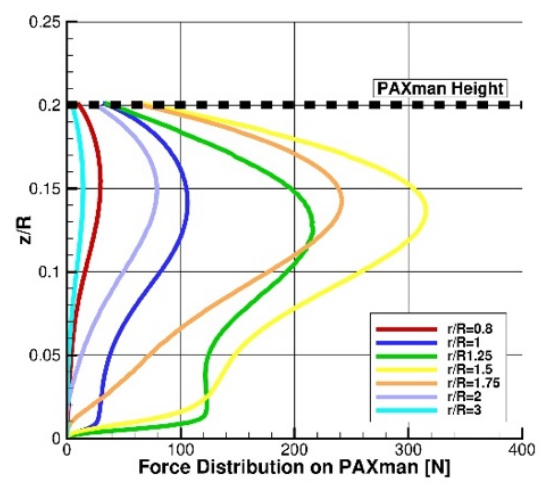

(c) Radial force distribution $f_{\mathrm{PAXman}}$ for medium weight aircraft

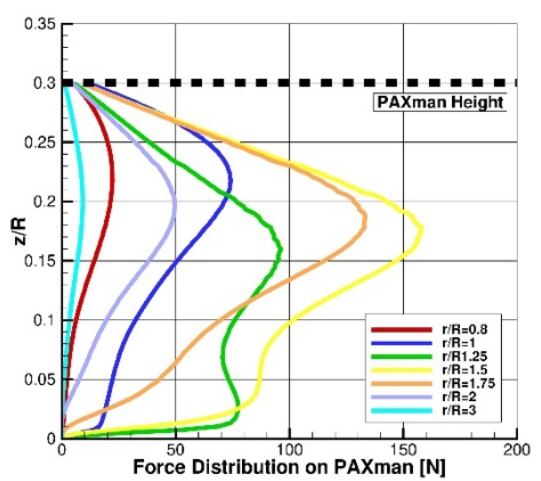

(b) Radial force distribution fPAXman for light weight aircraft

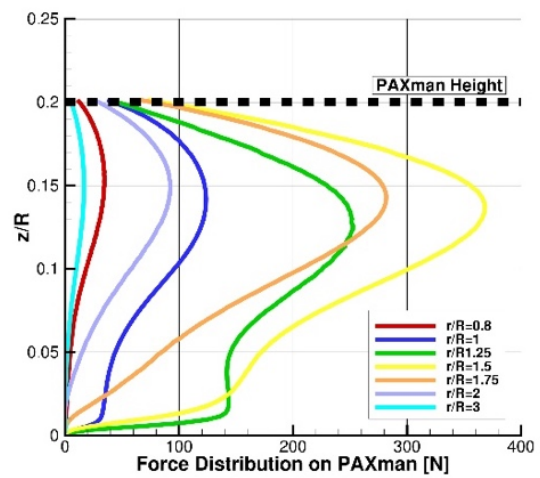

(d) Radial force distribution $f_{\mathrm{PAX} m a n}$ for heavy weight aircraft

Figure. 5 PAXman model forces calculated using the employed micro-rotor, scaled to $\mathrm{V}$ tip $\mathrm{m} / \mathrm{s}$. The micro rotor was operating at $\theta_{75}=12 \mathrm{deg}, \mathrm{Re}_{\text {tip }}=35000, \mathrm{M}_{\text {tip }}=0.08, \mathrm{~h} / \mathrm{R}=1$ and $\mathrm{C}_{\mathrm{T}}=0.03$. Scaling $\mathrm{V}_{\text {tip }}$ and $\mathrm{C}_{\mathrm{T}}$ for full scale rotor are listed in table 2 . 
In [24] the computed shear stress on the ground of the domain allowed to calculate the particle velocity uplift threshold. It was shown that the peak of the uplift ratio $\mathrm{u}_{t} / \mathrm{u}_{\mathrm{t}}$ is in proximity of $1 \mathrm{R}$ distance from the rotor. Using this information, it was possible to seed the ground with the particles that are more likely to be affected by the flow field. Figure 9 shows the evolution of the particles with time for two weight helicopter cases taken into account, with the rotor at two different heights above the ground $h / R=1$ and $h / R=0.5$. Different seeding positions have been defined between $1 \mathrm{R}$ and $1.5 \mathrm{R}$ in proximity of the ground level. Bombardment effect has not been considered for these simulations. These simulations involve about 100 revolutions, for a total time of 30 seconds. The flow field generated by the model rotor was not able to lift particles. As for the PAXman force computations, to obtain more realistic results the flow field has been scaled using a possible operational $\mathrm{V}$ tip for light and heavy weight helicopters considered in table 2 , and with the $\mathrm{C}_{\mathrm{T}}$ scaling factor. When the rotor is operating at $h / R=1$, the particles are uplifted by the flow field, and then move away from the rotor, following the radial direction. Particles are driven by the outflow that pushes them away from the rotor. However, depending on the strength of the outflow they reach different positions. Heavy weight helicopters have a stronger outflow, and in this case, particles can reach a maximum radial distance of $8.5 \mathrm{R}$, and a maximum height above the ground of $1 \mathrm{R}$. Once the particles reach the maximum altitude value, they fall again on the ground. The light weight helicopter case, shows lower values for maximum $h / R$ and $r / R$ $(0.75 \mathrm{R}$ and $6.5 \mathrm{R})$, however particles go further, the $3 \mathrm{D}$ separation criteria for wake encounters. On the other hand, when the helicopter is operating at $\mathrm{h} / \mathrm{R}=0.5$ particles show a different behaviour. Initially particles are uplifted from the ground, then two main branches spread, following different paths. Some of them, are re-ingested by the rotor, reaching the highest distance from the ground (around $2 \mathrm{R}$ for heavy weight test case and $1.75 \mathrm{R}$ for light weight). These particles can be dangerous for the crew and the aircraft. The rest of the particles keep following the radial direction far from the rotor and fall again on the ground (almost 7R for heavy weight helicopter and more than 6R for light). Particles that move away from the rotor can be dangerous for ground personnel and equipment, while the recirculation of the particles creates risks for the helicopter and the crew itself. During the re-ingesting phase particles can hit blade and fuselage and they create the cloud that degrades the visual of the pilot, creating a dangerous DVE condition.

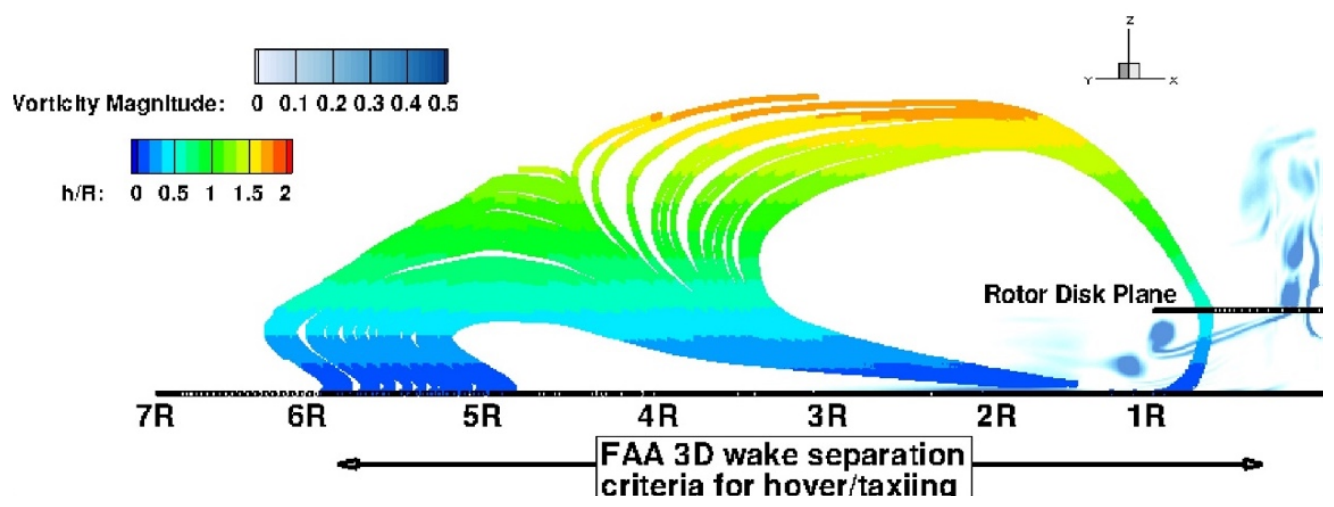

Figure. 6 Particle tracking, using velocities scaled with the light weight aircraft, tip velocity ( $\mathrm{V}$ tip = $220 \mathrm{~m} / \mathrm{s}$ ) and thrust coefficient for hovering at MTOW : $\mathrm{C}_{\mathrm{T}}=0.009$. The rotor was operating at $\theta_{75}=$ $12 \mathrm{deg}, \mathrm{Re}_{\text {tip }}=35000, \mathrm{M}_{\text {tip }}=0.08, \mathrm{~h} / \mathrm{R}=0.5$ and $\mathrm{C}_{\mathrm{T}}=0.035$. Contours correspond to the magnitude vorticity and the FAA 3 diameters separation distance is shown for comparison [5]. 


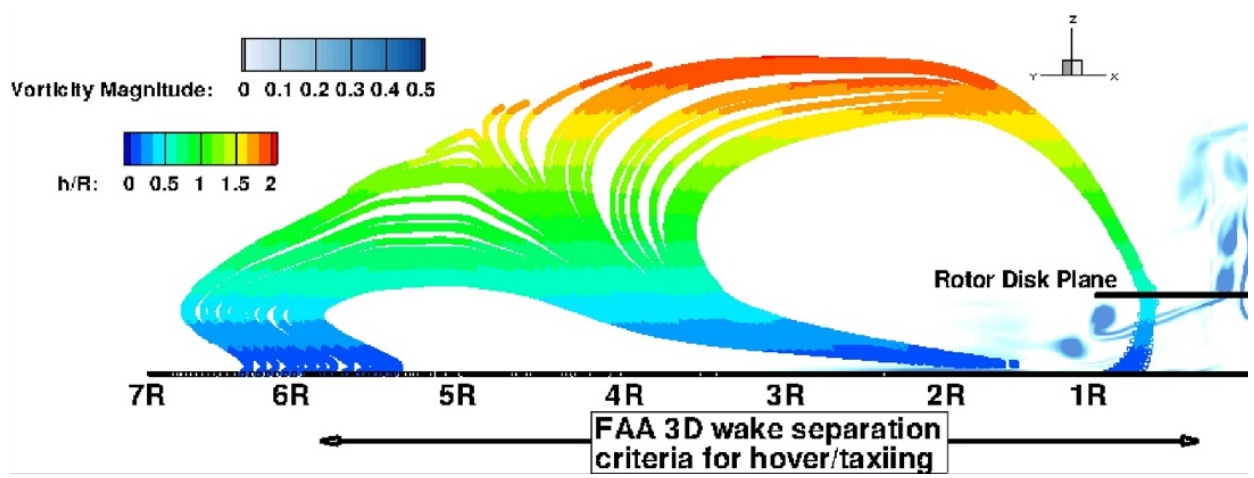

Figure. 7 Particle tracking, using velocities scaled with the heavy weight aircraft, tip velocity $(\mathrm{V}$ tip = $220 \mathrm{~m} / \mathrm{s}$ ) and thrust coefficient for hovering at MTOW : $\mathrm{C}_{\mathrm{T}}=0.017$. The rotor was operating at $\theta_{75}=$ $12 \mathrm{deg}, \mathrm{Re}_{\text {tip }}=35000, \mathrm{M}_{\text {tip }}=0.08, \mathrm{~h} / \mathrm{R}=0.5$ and $\mathrm{C}_{\mathrm{T}}=0.035$. Contours correspond to the magnitude vorticity and the FAA 3 diameters separation distance is shown for comparison [5].

h/R: $\quad \begin{array}{lllllll}0 & 0.15 & 0.3 & 0.45 & 0.6 & 0.75 & 0.9\end{array}$

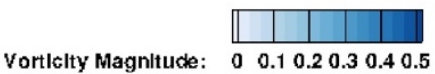

Rotor Plane Disk

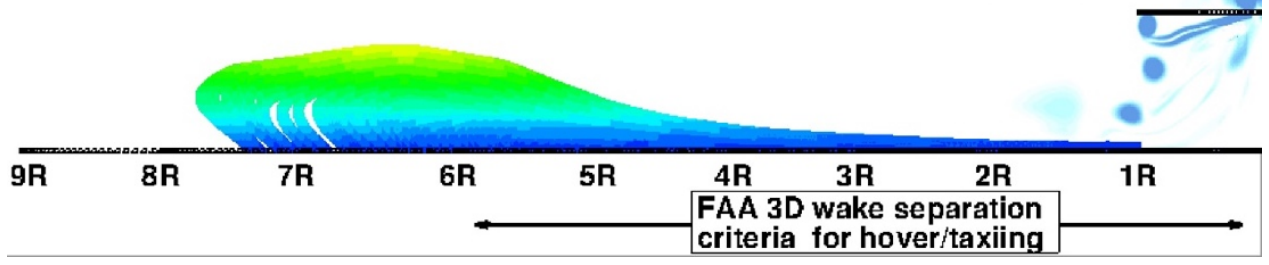

Figure 8. Particle tracking, using velocities scaled with the light weight aircraft, tip velocity ( $\mathrm{V}$ tip = $220 \mathrm{~m} / \mathrm{s}$ ) and thrust coefficient for hovering at MTOW : $\mathrm{C}_{\mathrm{T}}=0.009$. The rotor was operating at $\theta_{75}=$ $12 \mathrm{deg}, \mathrm{Re}_{\text {tip }}=35000, \mathrm{Mtip}=0.08, \mathrm{~h} / \mathrm{R}=1$ and $\mathrm{C}_{\mathrm{T}}=0.03$. Contours correspond to the magnitude vorticity and the FAA 3 diameters separation distance is shown for comparison [5].

h/R: $\quad \begin{array}{llllllll}0 & 0.15 & 0.3 & 0.45 & 0.6 & 0.75 & 0.9\end{array}$
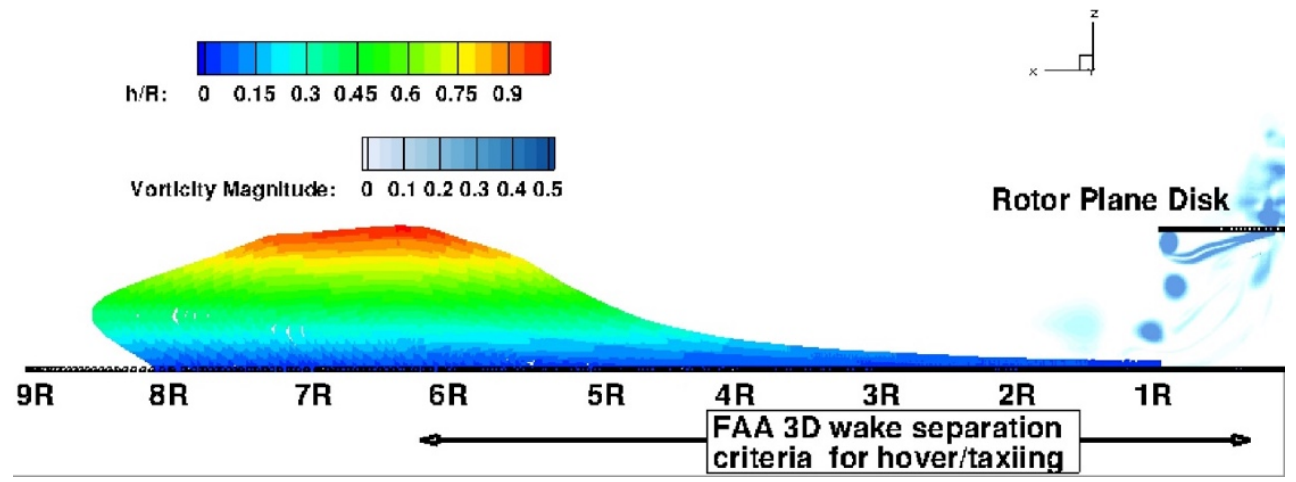

Figure. 9 Particle tracking, using velocities scaled with the heavy weight aircraft, tip velocity $(\mathrm{V}$ tip = $220 \mathrm{~m} / \mathrm{s}$ ) and thrust coefficient for hovering at MTOW : $\mathrm{C}_{\mathrm{T}}=0.017$. The rotor was operating at $\theta_{75}=12 \mathrm{deg} \mathrm{Re}_{\text {tip }} 35000, \mathrm{M}_{\mathrm{tip}}=0.08, \mathrm{~h} / \mathrm{R}=1$ and $\mathrm{C}_{\mathrm{T}}=0.03$. Contours correspond to the magnitude vorticity and the FAA 3 diameters separation distance is shown for comparison [5]. 


\section{Conclusions and future steps}

CFD results demonstrate fairly good agreement with the experimental data in terms of rotor performance and flow field, even with some differences in the outflow predictions for larger radial stations. The differences can be important if used to evaluate safety regions near the helicopter. Results employing different scaling approaches show different agreements with experiments, and only scaling with the hover induced velocity gives a complete understanding of the outflow analysis in terms of safety. The normalized magnitude of the radial velocity peak in the outflow and its position, related to PAXman height, are fundamental to obtain correct force estimations. In general, safety considerations show that the distance criterion based on the PAXman forces, can be deducted by the outflow analysis. Outflow forces after a distance of $3 \mathrm{R}$ are low for the three helicopter cases considered. Furthermore, for the lightest helicopter, there is no risk due to the outflow forces for ground personnel. It is clear that the wake encounter criterion of $3 \mathrm{D}$ can be adopted for ground operation, if the presence of particles on the ground can be excluded. A more detailed analysis shows that the forces obtained for the medium and heavy aircraft are high enough to be dangerous for personnel and equipment in an area between $1 \mathrm{R}$ and $2 \mathrm{R}$ away from the rotor, but after $3 R$ it is safer. The force distributions suggest that the chest is the part of the human body that is most influenced by the force due to the outflow, and this result can be part of the evaluation on definite safety regulations for people acting in proximity of the rotor. Particle tracking results show that particles can reach large distances away the rotor, exceed the limit of 3D. In general, it seems that the FAA limit for wake encounters cannot guarantee safety in presence of particles on the ground. It is also clear that to define a particle free zone, it is necessary to take into account the rotor operating conditions due to the strong influence of the disk loading and in general of the size of the aircraft on the particle paths. Particle paths are also strong influenced by the position of the rotor with respect to the ground. In this work, scaling factors are applied to a small-scale rotor to obtain full-scale PAXman forces and particle paths, however, the full physics of the brownout cannot be simulated in this way, due to the several factors. In general, the Reynolds number that is involved in the small and the full-scale scenario is dissimilar, leading to differences in the uplift phenomena and to a different evolution of the brownout cloud. This study can be a starting point for evaluating safe operational zones around a helicopter. A future step will consist of high detailed simulations to identify a proper safe zone from particles and compare it with other distance safety regulations like the 3 rotor diameters separation distance for wake encounters suggested in the Manual of Air Traffic Service [5] [6].

\section{Acknowledgments}

This project has received funding from the European Union's H2020 research and innovation programme under the Marie Sktodowska-Curie grant agreement No 721920

\section{Bibliography}

[1] D'Andrea, A., and Scorcelletti, F., "Enhanced numerical simulations of helicopter landing manoeuvres in brownout conditions," American Helicopter Society International 66th Annual Forum Proceedings, Phoenix, AZ, 2010.

[2] Taskgroup, R., "Rotary-wing brownout mitigation: technologies and training," Tech. Rep. RTO-TR-HFM-162, NATO Sci.Technol. Org., 2010.

[3] Mapes, P., Kent, R., and Wood, R., "DoD helicopter mishaps FY85-05: findings and recommendations," US Air Force, 2008. 
[4] Lee, T. E., Leishman, J. G., and Ramasamy, M., "Fluid dynamics of interacting blade tip vortices with a ground plane," Journal of the American Helicopter Society, Vol. 55, No. 2, 2010, pp. 22005-22005.

[5] CAA, "CAP 490: Manual of Air Traffic Services Part 1," 2015.

[6] Garcia-Dorado, I., Aliaga, D. G., Bhalachandran, S., Schmid, P., and Niyogi, D., "Fast Weather Simulation for Inverse Procedural Design of 3D Urban Models," ACM Transactions on Graphics (TOG), Vol. 36, No. 2, 2017, p. 21.

[7] Steijl, R., Barakos, G., and Badcock, K., "A framework for CFD analysis of helicopter rotors in hover and forward flight," International journal for numerical methods in fluids, Vol. 51, No. 8, 2006, pp. 819-847.

[8] Lawson, S., Woodgate, M., Steijl, R., and Barakos, G., "High performance computing for challenging problems in computational fluid dynamics," Progress in Aerospace Sciences, Vol. 52, 2012, pp. 19-29.

[9] Osher, S., and Chakravarthy, S., "Upwind schemes and boundary conditions with applications to Euler equations in general geometries," Journal of Computational Physics, Vol. 50, No. 3, 1983, pp. 447-481.

[10] Roe, P. L., "Approximate Riemann solvers, parameter vectors, and difference schemes," Journal of computational physics, Vol. 43, No. 2, 1981, pp. 357-372.

[11] Van Leer, B., "Towards the ultimate conservative difference scheme. V. A second-order sequel to Godunov's method," Journal of computational Physics, Vol. 32, No. 1, 1979, pp. 101-136.

[12] Jarkowski, M., Woodgate, M., Rokicki, J., and Barakos, G., "Towards consistent hybrid overset mesh methods for rotorcraft CFD," 2011.

[13] Steijl, R., and Barakos, G., "Sliding mesh algorithm for CFD analysis of helicopter rotor-fuselage aerodynamics," International journal for numerical methods in fluids, Vol. 58, No. 5, 2008, pp. 527-549.

[14] Silva, M., and Riser, R., "CH-47D tandem rotor outwash survey," AHS 67th Annual Forum, 2011.

[15] Preston, J. R., Troutman, S., Keen, E., Silva, M., Whitman, N., Calvert, M., Cardamone, M., Moulton, M., and Ferguson, S. W.,"Rotorwash Operational Footprint Modeling," Tech. rep., Missile Research Development and Engineergin Center Redstone Arsenal Al Missile Guidance Directorate, 2014.

[16] "Vertipedia," https://vertipedia.vtol.org/, Accessed: 23-09-2019.

[17] Alfred, J., Celi, R., and Leishman, J. G., "Flight Path Optimization for Brownout Mitigation Using a High-Fidelity Simulation Model," Journal of the American Helicopter Society, Vol. 62, No. 3, 2017, pp. 1-15.

[18] Wachspress, D., Whitehouse, G., Keller, J., Yu, K., Gilmore, P., Dorsett, M., and McClure, K., "A high fidelity brownout model for real-time flight simulations and trainers," 2009, pp. 278-301.

[19] Phillips, C., Kim, H. W., and Brown, R. E., "The flow physics of helicopter brownout," 66th American Helicopter Society Forum: Rising to New Heights in Vertical Lift Technology, 2010.

[20] Ghosh, S., Lohry, M. W., and Rajagopalan, R. G., "Rotor configurational effect on rotorcraft brownout," 28th AIAA Applied Aerodynamics Conference, 2010, p. 4238.

[21] Syal, M., Govindarajan, B., and Leishman, J., "Mesoscale sediment tracking methodology to analyze brownout cloud developments," 66th Annual Forum of the American Helicopter Society, Phoenix, AZ, 2010, pp. 11-13.

[22] Kutz, B., Günther, T., Rumpf, A., and Kuhn, A., "Numerical examination of a model rotor in brownout conditions," 2014, pp. 2450-2461.

[23] Tanner, P. E., "Photogrammetric characterization of a brownout cloud," 2011. 
[24] Rovere, F., Barakos, G., and Steijl, R., "CFD validation of a micro-rotor in grond effect," 45th European Rotorcraft Forum, CFAS, 2019.

[25] Kalra, T. S., Lakshminarayan, V. K., and Baeder, J. D., "CFD validation of micro hovering rotor in ground effect," American Helicopter Society 66th Annual Forum Proceedings, Citeseer, 2010.

[26] Hunt, J. C., Wray, A. A., and Moin, P., "Eddies, streams, and convergence zones in turbulent flows," 1988. 